\title{
Buchbesprechungen - Books Review - Livres Nouveaux
}

Lehmann, F.E.: Einführung in die physiologische Embryologic. 414 Seiten mit 132 Abbildungen. Verlag Birkhäuser, Basel 1946. Brosch. Fr. 34.-, gel), üanzleinen Fr. 38.-Die Entwicklungsphysiologie hat seit der Entdeckung des «OrganisatoГS» durch Hans Spcmann einen ungeahnten Aufschwung erlebt, indem zuerst mit operativen Melhoden und seit etwa zelin Jahren mit hochwertigen Stoffen die funktionellen Grundlagen der Formbildung untersucht wurden. Es sammelte sich cine ríesige Literatur an, und es entstand deshalb der Wunsch nach zusammenfassender Darstellung. Ich dart' auf das Buch von Spemann liinwei-sen, in dem in vollendeter Weise vor allem seine Lebensarbeit zusammen-gefaßt wird. Andere Bücher, wie díejcnígen von Huxley und De Deer (1934), von Paul Weiß (1939) oder Dalcq (1941), befassen sich mit den Problemen der Entwicklungsphysiologie, indem sie mehr von Hypothesen und theoreti-schen Ueberlegungen ausgehen, während die eigentlichen Experimenlalbefunde z. T. zu kurz kommen oder nur so $\Lambda$ veit berücksichtigt werden, $\lambda$ vie sie in das theorelische Gebäude hineinpassen. So isl es ein wirkliches Verdienst, wenn sich Lehmann der großen Mühe unlerzogen hat, eine Einführung in die physiologische Embryologic zu schreiben unter Zugrundelegung moderner Frage-stellung und unter Heranziehung des gesamlen heute bekannten experimentel-len Materials. Lehmann geht vom Objekt und seiner Eigenart aus und ist in seinem ganzen Buche bemiiht, das Entwicklungsgeschehen möglichst objekt-getreu zu erfassen. Er bespricht nur die Vorgänge, die sich in der sog. Primi-tiventwicklung bei Echinodermen und Amphibien abspielen, und läßt sich bei seiner Darstellung vom natürlichen Ablauf der Entwicklung vom Ei bis zur Ausbildung des embryonalen Grundplanes leiten. Diese Beschränkung auf die Primitiventwicklung und auf die beiden Untersuchungsobjekle Seeigelund Amphibienei hat den großen Vorteil, daß die ganze Beschreibung übersichtlich bleibt, hat aber den Nachteil, daß wíchtige Gebieíe der Entwicklungsphysiologie gar nicht zur Sprache kommen.

Das Buch zerfällt, seinem Inhalt entsprechend, in 3 Teile. In einer all-gemeinen Einleitung werden die nötigen Grundlagen geschaffen, auf welche sich dann die beiden folgenden Teile aufbauen. Für den Mediziner is! vor allem das Buch über die Entwicklungsphysiologie des Amphibieneies wertvoll, enthält es ja vor allem auch eine übersichtliche und ausführliche Darstellung des Organisatorproblems, wobei in einem besonderen Kapitel zu den Bezie-hungen von kernbedingten Störunge $\prod$ zu delerminaliveii und lopogenetischen Vorgängen der Frühentwicklung, in einem andern zu Organisator, Teratomen und Neoplasmen Stellung genommen wird. Besonders wertvoll empfindet der Leser, daß sich Lehmann nicht darauf beschränkt, Tatsachen zu beschreiben, sondern daß er sich bemiiht, Lücken aufzuweisen und auf neue Fragestellun-gen aufmerksam zu machen. Dadurch gewinnt das Buch nicht nur für den Außenstehenden, sondern auch für den Fachmann an Wert. Die Darstellungs-art ist klar und flüssig, die Abbildungen sind einfach gehalten, aber sehr gut verständlich; Druck und Ausstattung des Buches sind vorzüglich. Ich empfehle diese Einleitung in die physiologische Entwicklung alien biologisch Interes-

320 Buchbesprechungen - Books Review - Livres Nouveaux 
sierten aufs wärmsie und spreche gleichzeitig meine Hoffnung aus, daß durch dieses Buch die entwicklungsphysiologische Forschung auch innerhalb der Medizin den Raum gewinnen möge, der ihr zukommt. Töndury, Zurich. Bustinza-Lachiondo, F.: Les Antibiotiqucs Antimicrobicns et la Pénicilline. Edition Française par R. de Montmollin et M formules. A la Baconnière, Neuchâtel.

Les Editions de la Baconnière hatten eine glückliche Hand, als sie sich entschlossen, die Penicillin-Monographie von Bustinza-Lachiondo auch «Nichtspaniern» zugänglich zu machen und die Uebers < elzung Herrn und Frau Dr. de Mont mollin anzuvertrauen. Man wird sich ja zunächst fragen, wie es gelingt, nahezu 300 Seiten über Penicillin zu schreiben, vor allem, wenn die Chemie auf wenigen Seiten abgetan wird und auch der klinische Teil nicht viel mehr als 60 Seiten umfaßt. B. fand die Lösung, und zwar ohne auch nur einen Augenblick langweilig zu werden. Die historische Darstellung ist vorzüglich und läßt erkennen, wieviel besser man in Spanien über die Kriegsarbeit der Alliierten orientiert war als bei uns. Wenn B. in dankenswerter Weise die verschiedenen Methoden, die heute zur Penicillinauswertung im Gebrauch sind, so darstellt, daß sie ohne Schwierigkeiten übernommen werden können, so hat er andererseits sein Thema so weit gefaßt, daß Kliniker und biologische Chemike $\Gamma$ das Buch mit ebensoviel Interesse lesen werden wie chemotherapeutisch orientierte Bakteriologen. Im zweiten Kapitel gibt er eine kurze, im wesentlichen auf die neueste Literatur beschränkte Übersicht über die bakteriellen Fermente und Wuchsstoffe mit besonderer Berücksichtígung der Verhältnisse bei den Schimmelpilzen als seinem engeren Arbeitsgebiet. Der dritte Abschnitt ist der Chemolherapie, insbesondere den Sulfonamiden und ihrer Entwicklung, gewidmet. Auch hier werden vor allem die biologischen Probleme, wie Wirkungsmechanismus und sekundäre Resistenz, unter vorwiegender Berücksichtigung der anglo-amerikanischen Literatur dargestellt. Im vierten Abschnitt, der zum eigentlichen Penicillinkapitel überführt, gibt B. eine kurze, aber umfassende Darstellung aller übrigen Antibioticis, eine Darstellung, wie sie nur ein mit der Malerie restlos vertrauter Autor zu geben vermag. Die Penicillin kapitel selbst sind so vorzüglich geschrieben, daß jeder, der sich aus dem einen oder anderen Grund für P. interessiert, das Buch kennen muß, halt es doch in geschicktester Weise das Gleichgewicht zwischen großen Linien und minutiösen Details, die sonst nur in den uns z. T. immer noch nicht leicht zuganglichen Originalarbeiten zu finden sind. Durch einen Anhang, der die inzwischen freigegebenen chemischen Daten enthält, ist das Buch up to date gebracht. Die 83 Abbildungen sind z. T. technisch sehr illuslratív, z. T. geben sie Einblick in die pharmazeutisch-technischen Leislungen, welche die Engländer und Amerikaner während des Krieges auf diesem Gebiete vollbrachten, einzelne der nicht immer sehr schmeichelhaften Porträts erinnern allerdings stark an die südländische Affektlage des Verfassers. A. Grumbach. 